Classification

Physics Abstracts

$06.50-07.80-78.90-82.80$

\title{
EELS quantification near the single-atom detection level
}

\author{
Ondrej L. Krivanek(*), Claudie Mory, Marcel Tencé and Christian Colliex \\ Laboratoire de Physique des Solides, Bâtiment 510, Université Paris-Sud, 91405 Orsay, France
}

(Received March 29, 1991; accepted April 15, 1991)

\begin{abstract}
Parallel-detection electron energy loss spectrometers are able to detect the EELS signal originating from only a few atoms on thin substrates. The instrumental requirements for attaining this level of performance, and the methodology for quantifying the results are described. For the case of small thorium clusters on a thin carbon film, the detection limit with currently available instrumentation is shown to be one atom.
\end{abstract}

\section{Introduction.}

In 1975, Issacson and Johnson showed theoretically that detection limits attainable by electron energy loss spectroscopy (EELS) should be up to 3 orders of magnitude lower than by energy dispersive X-ray spectroscopy (EDXS) [1]. They also predicted that a single atom of fluorine embedded in a thin carbon film should be detectable by EELS. Their analysis was done for the case of a $100 \%$ efficient electron energy-loss spectrometer with parallel detection, and for the case of fluorine detection, they further postulated a probe of $0.3 \mathrm{~nm}$ diameter and $1 \mathrm{nA}$ current. Neither of these assumptions was realistic at the time, and the experimental spectra presented in their paper testified to the distance between the instrumentation then available and the predicted performance. Recent progress in instrumentation has covered much of the distance. This suggests that a reexamination of attainable detection limits should be both timely and interesting.

Two types of detection limits need to be considered [1]: the minimum detectable mass (MDM), which is the smallest amount of a pure element that can be detected, and the minimum detectable mass fraction (MMF), which is the smallest detectable concentration of an element homogeneously dispersed in a matrix composed of other materials. As shown in [1], MDM is critically dependent on the probe current density that can be delivered to the sample, whereas MMF depends primarily on the total current available in the probe. As a result, the lowest MMF is obtained with microscopes using thermionic electron guns able to deliver hundreds of $\mathrm{nA}$ to the sample [2]. In this paper, we concentrate on lowering the MDM as much as possible. The best microscopes currently available for this task typically have a cold field emission gun, and that is the experimental configuration employed here.

$\left.{ }^{\star}\right)$ Permanent address: Gatan R\&D, 6678 Owens Dr., Pleasanton, CA 94588, U.S.A. 
In the experimental section of this paper, it will become apparent that attaining the detection limits predicted by Isaacson and Johnson requires new methods of signal processing not envisaged by them. Finally, the demonstrated sensitivity of EELS will be compared with other microanalytical techniques, and probable future developments will be briefly discussed.

\section{Instrumentation.}

Figure 1 shows the instrumental arrangement used in this study. A parallel-detection electron energy loss spectrometer (Gatan PEELS model 666) is mounted on top of a Vacuum Generators HB501 scanning transmission electron microscope (STEM) equipped with a cold field-emission gun (FEG) operating at $100 \mathrm{keV}$ primary energy.

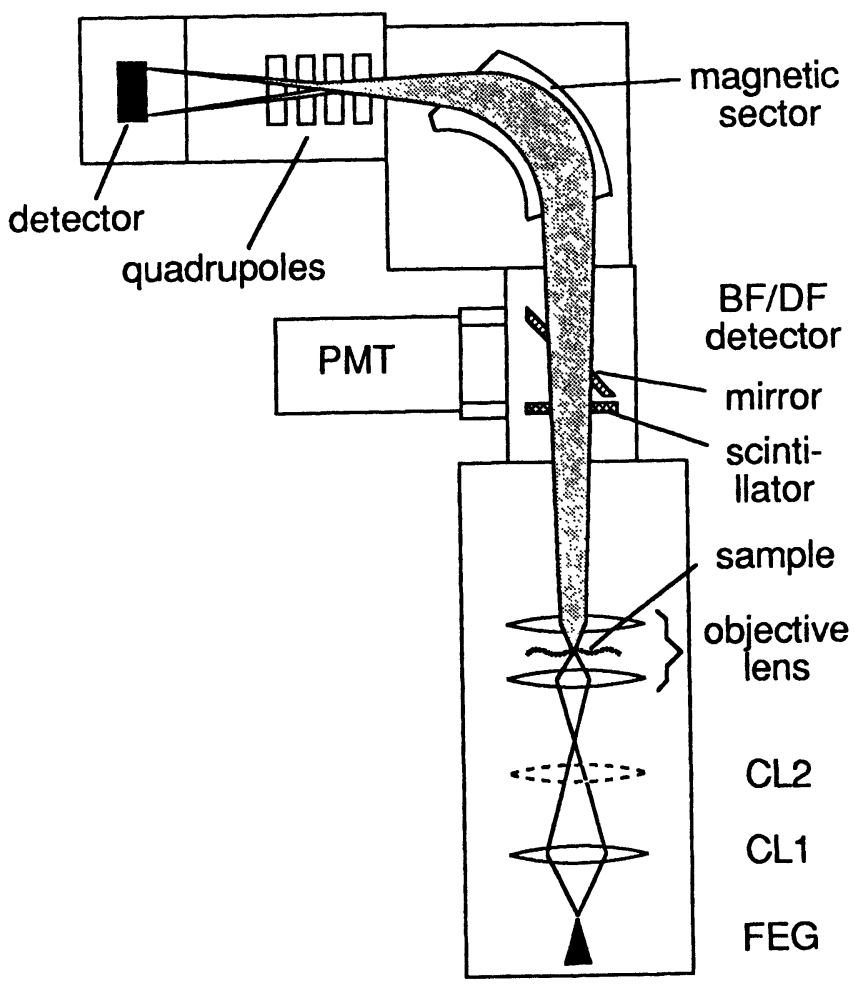

Fig. 1. - Schematic diagram of a parallel-detection electron energy-loss spectrometer (PEELS) mounted on a field-emission gun scanning transmission electron microscope (FEG-STEM), as used in this study.

The PEELS [3] consists of a single magnetic sector of $10 \mathrm{~cm}$ bending radius which is corrected for aberrations of 2 nd order, and produces an electron energy-loss spectrum with a dispersion of $1.8 \mu \mathrm{m} / \mathrm{eV}$ at $100 \mathrm{keV}$ primary energy. The spectrum is magnified by 4 quadrupole lenses, making the dispersion adjustable from $12.5 \mu \mathrm{m} / \mathrm{eV}$ to as high as $5 \mathrm{~mm} / \mathrm{eV}$. It is detected by a parallel detector consisting of 1024 channels each $2.5 \mathrm{~mm}$ wide and $25 \mu \mathrm{m}$ high, with a detective quantum efficiency which depends on the signal level, and is typically greater than 0.5 . The microscope 
[4] has an objective lens with $C_{\mathrm{s}}=3.1 \mathrm{~mm}$ and $C_{\mathrm{c}}=2.4 \mathrm{~mm}$, and is capable of forming a $1 \mathrm{~nm}$ diameter probe with about $1 \mathrm{nA}$ of current. In this study, it was typically operated with the second condenser lens switched off, and the first condenser and objective lenses adjusted to produce a beam of slightly less than $1 \mathrm{~nm}$ in diameter, and about $0.5 \mathrm{nA}$ beam current.

A detector module is placed between the PEELS and the microscope. It consists of a photomultiplier and three different scintillators any one of which can be moved into an on-axis position. Two of the scintillators are annular detectors designed for dark-field (DF) imaging, the third is a solid disk designed for bright-field (BF) imaging. The two DF scintillators have a central hole with a diameter of 2 or $4 \mathrm{~mm}$ respectively, lined by an aluminum tube. This allows the scintillators to also serve as the entrance aperture into the spectrometer, and minimizes any loss of a useful electron signal. The distance from the scintilator to the specimen is $290 \mathrm{~mm}$.

The post-specimen field of the microscope's objective lens compresses the range of angles of the electrons leaving the specimen by about $5 \times$, and makes the electrons arriving at the spectrometer appear as though they came from a crossover situated about $30 \mathrm{~mm}$ below the specimen plane. This gives an acceptance semi-angle of about $23 \mathrm{mrad}$ (at the specimen) when the $4 \mathrm{~mm}$ diameter DF scintillator is used, as was the case throughout these experiments.

Spectra were acquired and analyzed using Gatan EL/P software running on a Macintosh II computer.

\section{Experimental.}

In order to optimize the detection limit as much as possible, the specimens we have studied consisted of thorium-stained DNA and small thorium clusters dispersed on an ultra-thin carbon substrate. Thorium has the advantage of having an EELS edge with a large cross-section at an energy which is high enough to be clear of the low-loss region of an EELS spectrum, but is small enough to be below the carbon K-edge, so that the visibility of the thorium edge is not seriously affected by the presence of carbon. The specimens were prepared using standard biological staining techniques, and have been described previously $[5,6]$. Away from the heavily-stained DNA strands, the specimens contained thorium clusters whose size ranged down to about $0.5 \mathrm{~nm}$ diameter in annular DF images.

Figure 2 shows spectra from thorium atom clusters of three different sizes. Each spectrum was acquired with an acquisition time of 2.0 seconds, beam current of $0.5 \mathrm{nA}$, and the beam scanning rapidly over a sample area of 0.8 by $0.6 \mathrm{~nm}$ on top to the feature of interest. Scanning the beam meant that an image could be seen during the acquisition, and allowed us to check that the feature of interest remained in view. Drift of the sample was made unimportant by the short acquisition time used. The "small" cluster corresponded to a feature slightly less than $1 \mathrm{~nm}$ in diameter as seen in the DF image. A clear $\mathrm{Th}_{4,5}$ edge is visible for both the "large" and "medium" clusters, but there is no readily visible Th edge for the "small" cluster.

Figure 3 shows the three edges after background subtraction, made with a power-law fit over an energy interval spanning from 65 to $80 \mathrm{eV}$. The characteristic shape of the thorium $\mathrm{O}_{4,5}$ edge can be clearly distinguished for the "large" and "medium" clusters. In the case of the "small" cluster, the form of the edge is less distinct, but it is clear that there is an edge with a maximum at a similar energy as the edges from the other two clusters. For even smaller clusters, however, standard EELS quantification relying on background extrapolation and subtraction is clearly not going to be reliable.

Figure 4 shows first difference spectra from the medium and small clusters, computed numerically from the spectra shown in figure 2 . The computed first difference is the discrete equivalent of the first derivative of the spectrum intensity with respect to energy, $\mathrm{d} I / \mathrm{d} E$. It accentuates the 


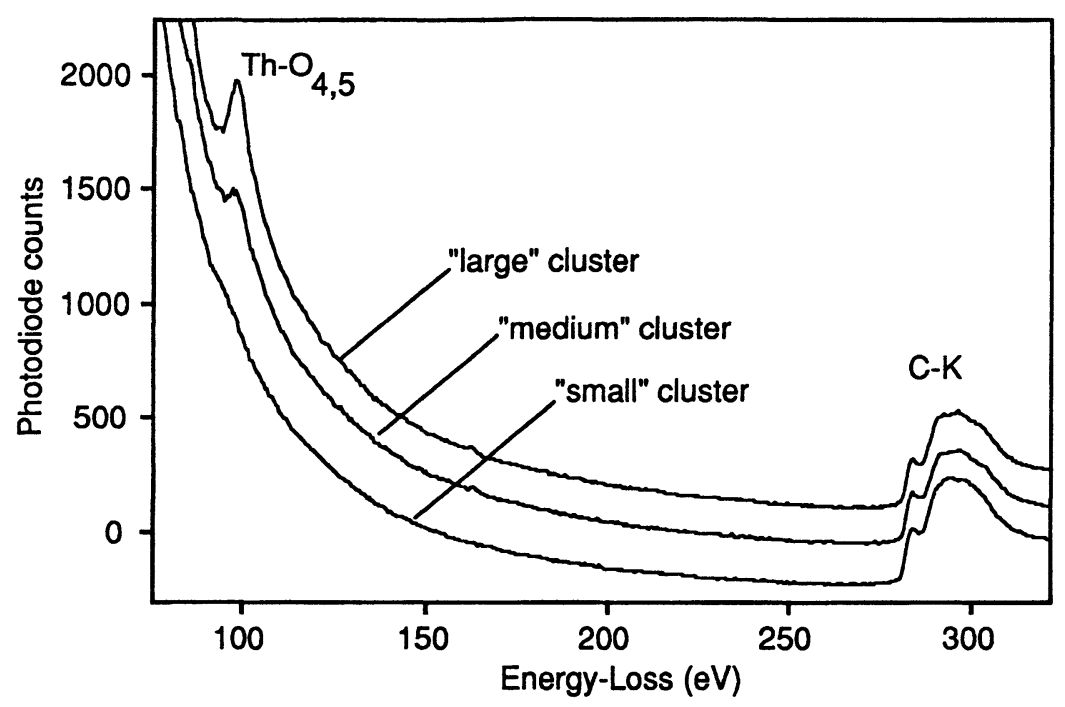

Fig. 2. - PEELS spectra obtained in 2 seconds each from three different clusters of thorium.

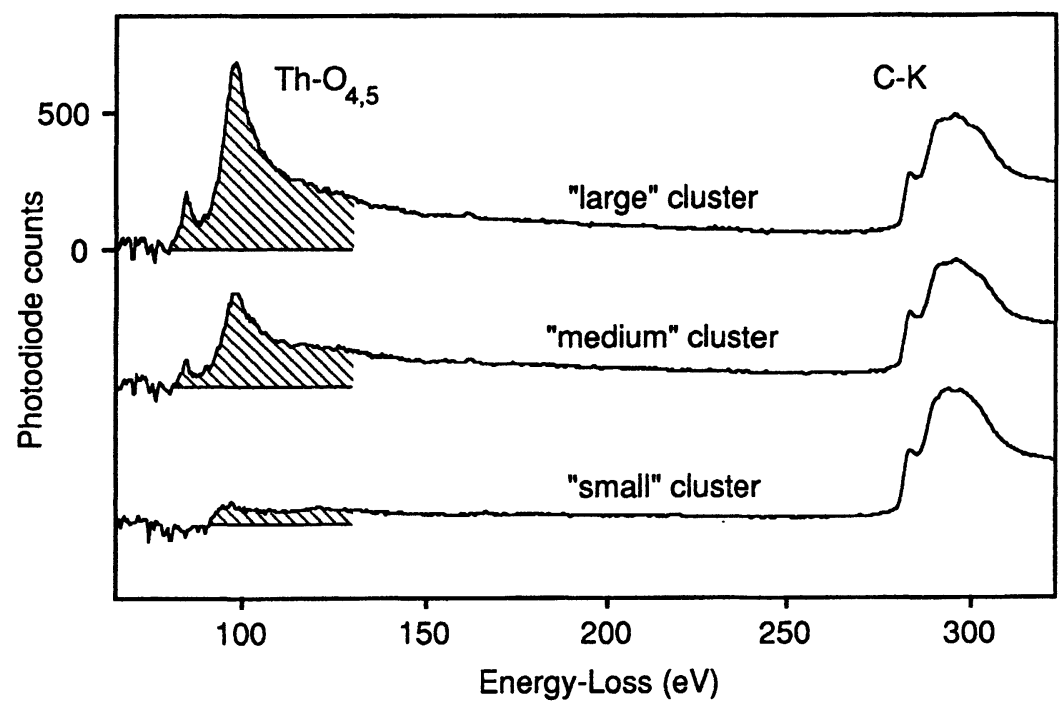

Fig. 3. - The spectra of figure 2 after subtraction of the background under the thorium edge. Shaded areas indicate net counts in the edges.

edges so that the weak Th edge visible in the spectrum from the medium cluster becomes a large feature, and the very weak edge in the spectrum from the small cluster also becomes readily visible. However, the computed first difference also accentuates spectral artifacts which are mostly due to the channel-to-channel gain variation of the parallel detector, such as the feature indicated by the small arrow in the "medium" cluster spectrum. This makes the computed difference unsuitable for detecting features even weaker than the Th edge in the "small" cluster spectrum. 


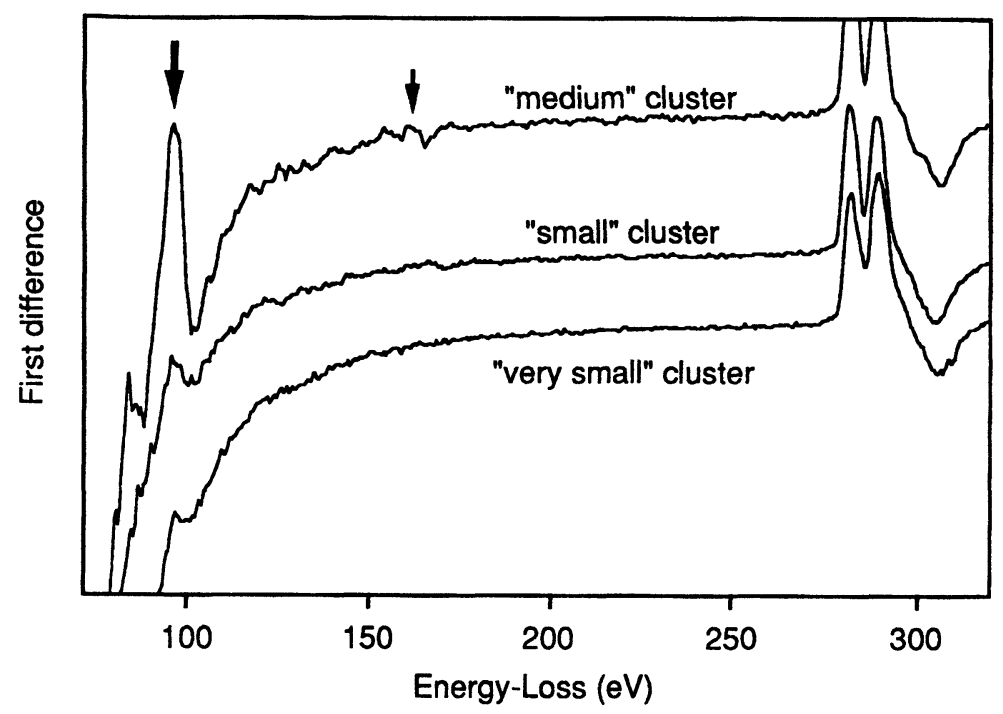

Fig. 4. - Two computed (top and middle) and one measured first difference spectra. The large arrow points to a peak due to the thorium $\mathrm{O}_{4,5}$ edge. The small arrow points to a peak due to the channel-to-channel gain variation.

The bottom spectrum in figure 4 is a first difference spectrum obtained from a "very small" cluster by recording a spectrum with a 2-second integration time, physically displacing the spectrum arriving at the detector by $5 \mathrm{eV}$ (by applying an extra voltage of $5 \mathrm{~V}$ to the drift tube leading through the PEELS magnetic sector), recording the spectrum a second time, and subtracting the two versions of the spectrum from each other. Such "measured" difference spectra have the advantage that artifacts due to the parallel detector remain in the same channel and therefore approximately subtract out, whereas real features in the spectrum move to a different channel of the detector, and are emphasized in the final result. The method is originally due to Shuman and co-workers [7]. The superiority of the measured difference spectra to computed ones is also demonstrated elsewhere in these proceedings [8]. Accordingly, the spectrum of a "very small" cluster shows much less systematic noise than the other two spectra. The weak feature visible at about the same energy as in the other two spectra confirms the presence of thorium.

Figure 5 shows a series of logarithmic difference spectra, each of which was obtained by dividing a first difference spectrum by an undifferentiated spectrum [8]. They have the advantage that they render the spectrum intensity away from the edge thresholds more constant and make it easier to compare the strength of weak features present in different spectra. Spectrum (a) was obtained from the computed "small" cluster difference spectrum shown in figure 4, spectra (b) to (e) were obtained from measured difference spectra. Spectra (b), (c) and (d) came from 3 different "very small" clusters, and (e) came from a specimen region away from any bright dots in the DF image.

\section{Spectrum quantification.}

The spectra of figures 2 and 3 are amenable to the standard method of EELS spectrum quantification [9]. Because the carbon K-edge is strong in the spectra and its cross-section is well known, 


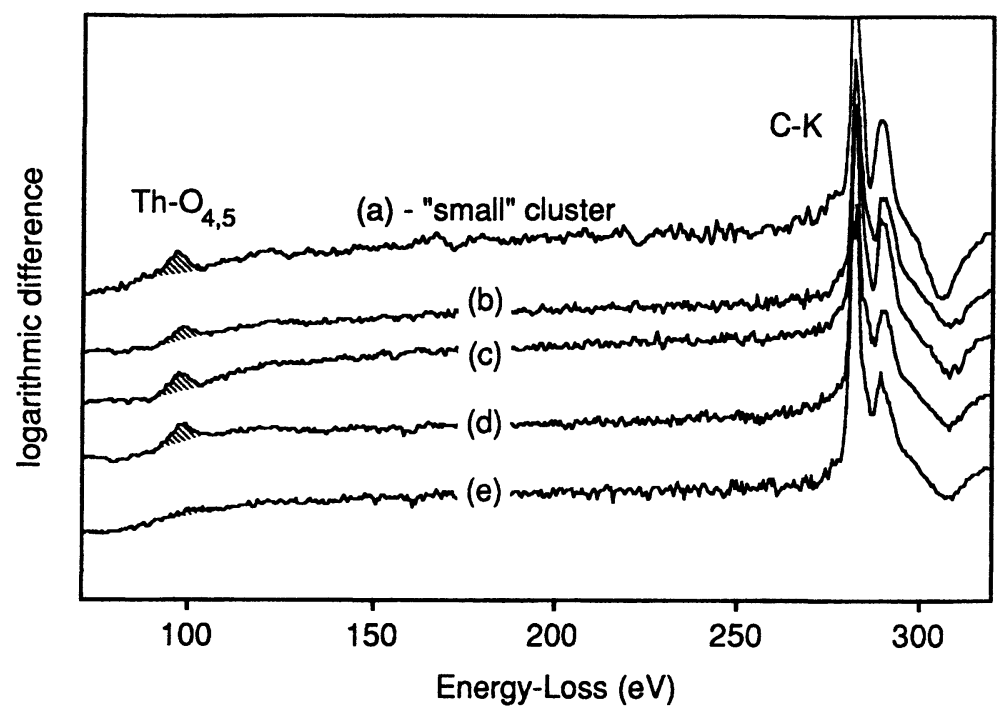

Fig. 5. - Logarithmic difference spectra of "small" (a) and "very small" (b-d) thorium clusters, and of the carbon film away from the clusters (e). The signal found at the thorium $\mathrm{O}_{4,5}$ edge threshold is indicated by the dashed areas.

we performed a relative quantification, and related the number of thorium atoms to carbon atoms using the standard expression

$$
\frac{N_{\mathrm{Th}}}{N_{\mathrm{C}}}=\frac{\sigma_{\mathrm{C}}(\beta, \Delta)}{\sigma_{\mathrm{Th}}(\beta, \Delta)} \frac{I_{\mathrm{Th}}(\beta, \Delta)}{I_{\mathrm{C}}(\beta, \Delta)}
$$

where $N$ stands for the number of detected atoms, $\sigma$ is the cross-section, $I$ the number of counts in the edge after background subtraction, and $\beta$ and $\Delta$ refer to the collection angle and the energy interval used.

The cross-sections of thorium and carbon were worked out using Rez's tables generalized oscillator strengths $[10,11]$ for a primary of $100 \mathrm{kV}$, a collection half-angle $\beta$ of $23 \mathrm{mrad}$ and an energy window $\Delta$ of $50 \mathrm{eV}$. They were $\sigma_{\mathrm{Th}}=1.80 \times 10^{-5} \mathrm{~nm}^{2}$, and $\sigma_{\mathrm{C}}=5.25 \times 10^{-7} \mathrm{~nm}^{2}$. (We give the cross sections in units of $\mathrm{nm}^{2}$ rather than the usual $\mathrm{cm}^{2}$ because it avoids having to work with unnecessarily large exponents). This means that the thorium cross-section was 34 times larger than the carbon cross-section, and confirms that thorium is an ideal candidate for single-atom detection.

Table I shows the intensities in the first $50 \mathrm{eV}$ of the thorium and carbon edges in each spectrum after background subtraction, the intensity ratios, the resultant thorium-to-carbon atomic ratio, and the absolute number of thorium atoms. To obtain the number of thorium atoms with the relative quantification method, the number of carbon atoms within the sample volume probed for thorium needs to be known. There are several ways of determining this number, such as measuring the weight of the carbon film at the time of its deposition with a quartz crystal monitor, or performing an absolute EELS quantification using the carbon K-edge. The method employed here was to determine the film thickness from the ratio of the total inelastic signal to the zero loss peak intensity (Ref. [9], pp. 291-297). This is done simply by recording a low loss spectrum containing an unsaturated zero loss peak, calibrating the spectrum in energy, and calling up the thickness-measuring routine of EL/P. The routine automatically separates the zero loss peak from 
the rest of the spectrum, and works out the thickness of the film in terms of the mean free path $\lambda_{\mathrm{i}}(\beta)$ for all inelastic scattering falling within the spectrometer acceptance angle. Since plasmon scattering is the predominant inelastic scattering event, and the $23 \mathrm{mrad}$ collection angle used here was much larger than the characteristic angle for plasmon scattering, we took $\lambda_{\mathbf{i}}(\beta)$ to be equal to the mean free path for plasmon scattering in carbon given in [12] as $\lambda_{\mathrm{p}}=62 \mathrm{~nm}$. The thickness value worked out by EL/P was $0.07 \lambda_{\mathrm{i}}$, corresponding to a film thickness of $t_{\mathrm{C}}=4 \mathrm{~nm}$.

Table I. - Quantification of the spectra of figures 2 and 3. See text for how the values were derived.

\begin{tabular}{|l|c|c|c|c|c|}
\hline \multicolumn{1}{|c|}{ spectrum } & $\begin{array}{c}\text { counts in } \\
\text { Th } \mathrm{O}_{4,5} \text {-edge } \\
(\Delta=50 \mathrm{eV})\end{array}$ & $\begin{array}{l}\text { counts in } \\
\mathrm{C} \mathrm{K} \text {-edge } \\
(\Delta=50 \mathrm{eV})\end{array}$ & $I_{\mathrm{Th}} / I_{\mathrm{C}}$ & $N_{\mathrm{Th}} / N_{\mathrm{C}}$ & $N_{\mathrm{Th}}$ \\
\hline "large" cluster & 27428 & 27330 & 1.00 & 0.029 & 23 \\
"medium" cluster & 16619 & 26667 & 0.62 & 0.018 & 14 \\
"small" cluster & 5093 & 29398 & 0.17 & 0.005 & 4 \\
\hline
\end{tabular}

The number of carbon atoms in the volume probed for thorium is $N_{\mathrm{C}}=\rho_{\mathrm{C}} t_{\mathrm{C}} A$, where $\rho_{\mathrm{C}}$ is the density of the carbon film expressed in atoms per $\mathrm{nm}^{3}$, and $A$ is the area of the sample probed for thorium. We take $\rho_{\mathrm{C}}$ to be 100 atoms per $\mathrm{nm}^{3}$ (which corresponds to carbon of specific weight of $2 \mathrm{~g} / \mathrm{cm}^{3}$ ). The area $A$ is probably the hardest number to determine accurately in the present quantification, since it must take account of the intensity contained in the tails of the electron probe, of beam spreading in the foil, and of the broadening of the thorium signal due to delocalization of inelastic scattering. It must also include the 0.8 by $0.6 \mathrm{~nm}$ area over which the beam was scanning. Beam spreading in a $4 \mathrm{~nm}$ thick foil is less than $0.1 \mathrm{~nm}$ for a beam of $23 \mathrm{mrad}$ angular spread, but delocalization [13] of the Th ionization event (the ability of a fast electron to ionize the thorium $O$ shell even though it is passing some distance away from the thorium atom) amounted to probably about 0.5 to $1 \mathrm{~nm}$ [14]. We take the basic area covered by the electron probe to be $1 \mathrm{~nm}^{2}$, and assume that delocalization and probe tails broadened it to $2 \mathrm{~nm}^{2}$ effective area.

Using the values above gives 800 as the number of carbon atoms in the sample volume probed for thorium, and leads to the absolute numbers of thorium atoms in the three clusters given in the last column of table I. The uncertainty in $A$ means that the number of thorium atoms should probably be taken with an error bar of $+100,-50 \%$. Even so, it is clear that the "small" cluster contained less than 10 atoms of thorium.

An alternate way of determining the number of thorium atoms is to do an "absolute" quantification, using:

$$
N_{\mathrm{th}}=\frac{I_{\mathrm{th}}(\beta, \Delta)}{\sigma_{\mathrm{th}}(\beta, \Delta)} \frac{A}{\xi \cdot n_{\mathrm{e}^{-}}},
$$

where $\xi$ is the detector conversion efficiency (the number of counts registered by the computer per one detected electron), and $n_{\mathrm{e}^{-}}$is the number of electrons detected in the whole spectrum (including the zero loss peak). $n_{\mathrm{e}^{-}}$is conveniently measured using the shield current picoammeter of the PEELS, and was $6.2 \times 10^{9}$ electrons ( $0.5 \mathrm{nA}$ for 2 seconds). The conversion efficiency of the PEELS used was 0.040 (25 electrons per one computer count), and we again take $A$ to be $2 \mathrm{~nm}^{2}$. Using equation (2) and the number of counts listed in table I then gives the number of 
thorium atoms in the small cluster as 2.3 . This is almost $2 \times$ less than the number determined by relative quantification. The difference can be due to several causes: a) part of the EELS spectrum not being detected by the photodiode array due to poor alignment, b) the correct value of the collection angle, the detector conversion efficiency, or the current detected by the picoammeter being smaller than the values used here, c) the correct absolute values of both the thorium and carbon cross-sections being smaller than the values used here (if only the thorium value were in error, then the result of the relative quantification would change also, and the discrepancy would remain), and d) the thickness determination using the published value of $\lambda_{p}$ being in error (film thinner than we determined), for instance because we neglected the contribution of the surface plasmon. We intend to investigate the cause of the discrepancy in more detail in the future. For the present, we can use it as an indication of the likely error due to factors other than the uncertainty in $A$.

Table II compares the intensity of the feature detected at the $\mathrm{Th}_{4,5}$ energy in the various logarithmic first difference spectra of figure 5. The thorium peak intensities were obtained by subtracting straight-line fits under the peaks as shown by the shaded areas in the figure. The $\mathrm{C}$ peak intensity, which was used for normalization, was obtained by integrating the intensity under the double peak feature extending from about 280 to about $330 \mathrm{eV}$, again after the subtraction of a straight-line background fit. The last column in the table gives the absolute number of the Th atoms detected using 4 as the number of atoms in the "small" cluster. Had we used 2.3 atoms as determined by the absolute quantification, the number of thorium atoms would have come out as about 1,2 , and 2 for spectra (b), (c), and (d), respectively.

Table II. - Quantification of the logarithmic difference spectra of figure 5.

\begin{tabular}{|l|c|c|c|c|}
\hline spectrum & $\begin{array}{c}\text { counts in } \\
\text { Th peak }\end{array}$ & $\begin{array}{c}\text { counts in } \\
\text { C peak }\end{array}$ & $\begin{array}{c}\text { normalized } \\
\text { Th counts }\end{array}$ & $\begin{array}{c}\text { number of } \\
\text { Th atoms }\end{array}$ \\
\hline a("small" cluster) & 1214 & 18442 & 1214 & $(4)$ \\
b & 711 & 22270 & 588 & 1.9 \\
c & 1198 & 22099 & 999 & 3.3 \\
d & 1038 & 19966 & 957 & 3.1 \\
e & 162 & 15629 & 189 & 0.6 \\
\hline
\end{tabular}

The number of atoms in the last column of table II was worked out assuming a linear relationship between the area under the peak in the logarithmic difference spectrum, and the number of atoms. This would not hold for large clusters giving a significant signal in the undifferentiated spectrum. However, in the present case the thorium signal was so weak in the normal spectrum that the logarithmic derivatives were obtained by dividing the difference spectra by essentially the same normal spectrum, thus guaranteeing that the logarithmic difference signal strength was linearly related to the first difference signal which is in turn linearly related to the edge intensity in the normal spectrum. Provided that there were no major changes in the edge fine structure which depended on the number of atoms in the clusters, the logarithmic difference signal strength was therefore linearly related to the number of atoms, and our assumption was valid. 


\section{Discussion.}

The various quantification methods employed above, together with the uncertainty in $A$, show that the minimum number of atoms of Th detected when the probe was placed on a bright dot in the DF image was between 1 and about 4.

An even more interesting result is the fraction of an atom (0.6) entered in table II for spectrum (e), which should show no thorium. A fraction of an atom might be correctly detected if, for instance, the probe was placed close to, but not on top of, an isolated atom, or the atom jumped away during the acquisition.

In order to determine the statistical significance of this intriguing result, we estimated the r.m.s. noise in a single channel of the spectrum near the thorium peak by subtracting a smoothed version (obtained with an 11-point smoothing function) of the spectrum from the original spectrum, squaring the difference, summing the squared difference over $10 \mathrm{eV}$ wide energy intervals just below and just above the thorium peak, dividing the total by the number of channels in the two energy intervals, and taking the square root. The noise in the background-subtracted thorium peak was then estimated by multiplying the noise in a single channel by the square root of the total number of channels in the thorium peak plus the two adjacent energy intervals that were used to fit the background under the peak. This procedure gave a noise content in the subtracted thorium peak as about 20 counts, indicating that the signal-to-noise ratio for the weak peak in spectrum (e) was of the order of 10 , and the peak was therefore statistically significant. It thus appears that there was actually some thorium signal even in the "no-thorium" spectrum, probably from atoms a few nm away being illuminated by electrons in the probe tail. We note that attaining a detection limit of $a$ fraction of an atom is a necessary prerequisite to being able to reliably detect a single atom. The fact that this limit has been reached means that single-atom microanalysis by EELS is now a reality.

Apart from the discrepancy between the absolute and the relative quantification methods, by far the largest source of uncertainty in the present work has been the effective size of the sampled area. Its accurate determination will require a precise characterization of the size and shape of the electron probe (including probe "tails"), and a precise knowledge of the magnitude of the delocalization.

One possible approach to decreasing the importance of the precise shape of the probe tails is to scan a larger part of the sample during the acquisition than the 0.6 by $0.8 \mathrm{~nm}$ used here. If the scanned area is larger than both the probe and the possible delocalization, the relative uncertainty in the total probed area is much reduced. This approach has been used by Leapman and coworkers in their work on biological samples $[15,16]$, in which the location of the atoms of interest within a relatively large biological molecule is typically not known precisely. The disadvantage of the approach is that the signal from an isolated atom or a group of atoms is reduced as one over the scanned area size. However, if the feature of interest is visible in the DF image which can be acquired simultaneously with the EELS spectrum, this approach permits long recording times by making it possible to bring the feature back into view if it starts drifting away, and to check that it has not been destroyed by radiation damage.

The number of carbon atoms in the $2 \mathrm{~nm}^{2}$ area probed for thorium was about 800 . Hence detecting a single atom of thorium also meant detecting thorium in a concentration of around 0.1 atomic \%. This largely explains why the thorium signal was nearly invisible in undifferentiated spectra from the "small" and "very small" clusters. In general, detecting extremely small absolute quantities of matter will also automatically mean having to attain a small MMF, especially if the sample thickness is nearer the typical 20 to $50 \mathrm{~nm}$, rather than $4 \mathrm{~nm}$ as here. Minimizing the probe size and using higher energy edges which suffer less from delocalization will make the MMF demands less severe. Even so, it is likely that detecting fewer than 10 atoms will typically only be 
possible with the measured difference spectra which diminish the importance of the channel-tochannel gain variation of the parallel detector.

We note that the objective lens of the microscope used in this study only attains a $C_{\mathbf{s}}$ figure $(3.1 \mathrm{~mm})$ that was available in CTEM instruments more than 20 years ago, and that improving the spherical aberration by a factor of 3 should improve the visibility of heavy atoms such as thorium in the dark field images considerably. Because of delocalization, the resultant lowering of the detection limit for thorium using the $\mathrm{Th}^{\mathrm{O}} \mathrm{O}_{4,5}$ edge will be less pronounced, but there will be larger improvements for edges at higher energy.

The radiation resistance of an atom which is being ionized many times over, and yet manages to remain under the beam during the time of observation, is a separate and vast topic. Heavy atoms such as thorium and uranium seem to be especially suitable in this regard [17], and cooling the specimen to liquid nitrogen temperatures may also be helpful. Cooling is in any case highly advantageous because it allows one to work with a wide range of specimens without having to worry about contamination build-up under the electron beam [18].

\section{Conclusion.}

Single atoms of elements with favorable cross-sections such as thorium or uranium can now be detected in a few seconds using a PEELS mounted on a STEM capable of delivering a beam current of a few hundred pA into a sub-nm probe. The signal from a single atom is typically not identifiable in an undifferentiated spectrum. However, it becomes distinguishable in "measured" difference spectra obtained by shifting spectra on the parallel detector, and subtracting them. Quantifying the difference spectra is currently only possible by comparing them to difference spectra from larger clusters of about 10 atoms or more, whose size can be determined using conventional EELS quantification methods.

The ultimate demonstration of the capability to detect single atoms will probably come from an experiment showing clear plateaus in the detected inner-shell intensity corresponding to $0,1,2$ etc. atoms. The practical applications of the available sensitivity do not have to await such a tour de force, but can instead concentrate on studying materials in which the detection of extremely small clusters of atoms giving a clear EELS signal is of vital importance. Here we point to the pioneering work of Leapman and co-workers $[15,16]$, who are exploring numerous interesting problems of this type from the field of biology.

The ability of EELS to analyze a single atom while leaving it in its surroundings distinguishes it from other techniques such as time-of-flight field-ion spectroscopy (atom-probe), which has been able to detect and analyze single atoms for some time [19], but only once they have been desorbed from a sharp tip. It also gives a marked advantage to EELS over scanning tunneling spectroscopy, which has no element-specific signal with which to work.

In the future, the domain of single-atom microanalysis will no doubt be extended to many atoms other than thorium and uranium. However, the degree of difficulty will be inversely related to the size of the atom's cross-section. We note that whereas the thorium $\mathrm{O}_{4,5}$ cross-section is 34 times larger than the carbon $\mathrm{K}$-edge one (in a $50 \mathrm{eV}$ wide window with a 23 mrad collection angle at $100 \mathrm{kV}$ ), the fluorine $\mathrm{K}$ cross-section is 7 times smaller than the carbon one. Singleatom microanalysis for the case originally discussed by Isaacson and Johnson, fluorine in a carbon matrix, may therefore still be some distance away. 


\section{Acknowledgements.}

We are grateful to Dr. E. Delain of Institute G. Roussy for providing us with the samples, and to Prof. P. Rez of Arizona State University and Dr. M.K. Kundmann of Gatan R\&D for supplying us with the thorium and carbon cross-sections.

\section{References}

[1] ISAACSON M. and JOHSON D., Ultramicroscopy 1 (1975) 33.

[2] KRIVAneK O. L. and Kundmann M. K., Proc. 49th EMSA meet. G. W. Bailey Ed. (San Francisco Press, San Francisco, 1991) to be published.

[3] KRIVANEK O. L., AHN C. C. and KEENEY R. B., Ultramicroscopy 22 (1987) 103.

[4] MORY C., TENCÉ M. and CollieX C., J. Microsc. Spectr. Electron. 10 (1985) 381.

[5] MORY C. et al., Ultramicroscopy 7 (1981) 161.

[6] MORY C. and COllieX C., Ultramicroscopy 28 (1989) 339.

[7] SHUMAN H. and KRUIT P., Rev. Sci. Instrum. 56 (1985) 231.

[8] KundMANN M. K. and KRIVANEK O. L., these proceedings.

[9] EgeRTon R. F., Electron Energy-Loss Spectroscopy in the Electron Microscope (Plenum Press, New York and London, 1986) pp. 262-266.

[10] ReZ P. and LEAPMAN R. D., Analytical Electron Microscopy 1981, R. H. Geiss Ed. (San Francisco Press, 1981) p.181.

[11] Leapman R. D., Rez P. and Meyers D. F., J. Chem. Phys. 72 (1980) 1232.

[12] RAETHER H., Excitation of Plasmons and Interband Transitions by Electrons (Springer-Verlag, Berlin, 1980) p. 41.

[13] MoRy C., KoHL H., Tencé M. and CollieX C., Ultramicroscopy, to be published (1991).

[14] Colliex C., Krivanek O. L. and Trebila P., Inst. Phys. Conf. Ser. 61 (1981) 183.

[15] LEAPMAN R. D. and ANDREWS S. B., J. Microsc., to be published, and these proceedings.

[16 LEAPMAN R. D. and HUNT J., these proceedings.

[17] ISAACSON M., OHTSUKI M., and UTLAUT M., Introduction to Analytical Electron Microscopy, J. J. Hren, J. I. Goldstein and D. C. Joy Eds. (Plenum Press, New York and London, 1979) p. 343.

[18] HREN J. J., Introduction to Analytical Electron Microscopy, J. J. Hren, J. I. Goldstein and D. C. Joy Eds. (Plenum Press, New York and London, 1979) p. 481.

[19] MÚller E. W., Panitz J. A. and McLaue S. B., Rev. Sci. Instrum. 39 (1968) 83. 